\title{
Cathepsin B inactivation attenuates hepatic injury and fibrosis during cholestasis
}

\author{
Ali Canbay, ${ }^{1}$ Maria Eugenia Guicciardi, ${ }^{1}$ Hajime Higuchi, ${ }^{1}$ Ariel Feldstein, ${ }^{1}$ \\ Steven F. Bronk, ${ }^{1}$ Robert Rydzewski, ${ }^{2}$ Makiko Taniai, ${ }^{1}$ and Gregory J. Gores ${ }^{1}$ \\ ${ }^{1}$ Division of Gastroenterology and Hepatology, Mayo Medical School, Clinic, and Foundation, Rochester, Minnesota, USA \\ ${ }^{2}$ Celera Genomics, South San Francisco, California, USA
}

\begin{abstract}
Although a lysosomal, cathepsin B-dependent (Ctsb-dependent) pathway of apoptosis has been described, the contribution of this pathway to tissue damage remains unclear. Our aim was to ascertain if Ctsb inactivation attenuates liver injury, inflammation, and fibrogenesis after bile duct ligation (BDL). In 3-day BDL mice, hepatocyte apoptosis, mitochondrial cytochrome $c$ release, and serum alanine aminotransferase (ALT) values were reduced in $\mathrm{Cts}^{-/-}$versus $\mathrm{Cts} b^{+/+}$animals. Likewise, R-3032 (a Ctsb inhibitor) also reduced these parameters in BDL WT mice. Both genetic and pharmacologic inhibition of Ctsb in the BDL mouse reduced (a) hepatic inflammation, as assessed by transcripts for CXC chemokines and neutrophil infiltration, and (b) fibrogenesis, as assessed by transcripts for stellate cell activation and sirius red staining for hepatic collagen deposition. These differences could not be ascribed to alterations in cholestasis. These findings support a prominent role for the lysosomal pathway of apoptosis in tissue injury and link apoptosis to inflammation and fibrogenesis. Ctsb inhibition may be therapeutic in liver diseases.
\end{abstract}

J. Clin. Invest. 112:152-159 (2003). doi:10.1172/JCI200317740.

\section{Introduction}

Liver injury and fibrosis are cardinal features of most chronic human liver diseases. These two separate, nefarious processes combine to cause end-stage liver disease with cirrhosis and liver failure. Current therapeutic options for liver diseases, unfortunately, remain inadequate, and, as a result, the social economic burden of liver diseases remains high $(1,2)$. Further insights into the mechanisms contributing to liver injury and fibrosis and the signals potentially linking these two disease processes are necessary to help develop additional and effective rational therapies.

The mechanisms of liver cell injury are complex and involve the interplay of cytokines, toxic bile acids, reactive oxygen species, inflammatory cells, and alterations in vascular flow. The interdependent, multifaceted mechanisms culminating in liver injury have made identification of dominant processes difficult. Recent investigations have demonstrated that hepatocyte apoptosis, however, is a nearly ubiquitous response of the liver to injury (3).

Received for publication January 2, 2003, and accepted in revised form May 6, 2003.

Address correspondence to: Gregory J. Gores, Mayo Medical School, Clinic, and Foundation, 200 First Street SW, Rochester, Minnesota 55905, USA. Phone: (507) 284-0686;

Fax: (507) 284-0762; E-mail: gores.gregory@mayo.edu.

Conflict of interest: The authors have declared that no conflict of interest exists.

Nonstandard abbreviations used: alanine aminotransferase (ALT); bile duct ligation/ligated (BDL); cathepsin B (Ctsb); cathepsin knockout $\left(\mathrm{Ctsb}^{-/-}\right)$; WT $\left(\mathrm{Ctsb}^{+/+}\right)$; hepatic stellate cell (HSC); chemokine (CXC) ligand 1 (KC); macrophage inflammatory protein-2 (MIP-2); myeloperoxidase (MPO); $\alpha$-smooth muscle actin ( $\alpha$-SMA); collagen $\alpha 1$ (I) (COL1A1); tissue inhibitor of metalloproteinases (TIMP).
In addition to apoptosis, virtually all liver diseases are associated with an inflammatory response. The interplay between hepatocyte apoptosis and inflammation is likely complex, but recent concepts suggest a potential mechanistic relationship between these two processes. Apoptosis has been shown to induce CXC chemokines in the liver, potent chemotactic agents for neutrophils (4). Consistent with these data, inhibition of hepatocyte apoptosis blocks neutrophil transmigration into the liver during injurious conditions (5-8). Inhibition of hepatocyte apoptosis may therefore be anti-inflammatory in hepatic diseases, although this concept requires further examination.

TNF- $\alpha$ and toxic bile acids have been implicated in liver injury and hepatocyte apoptosis, especially during cholestasis (a pathophysiologic condition induced by impaired bile formation) (9-12). Several studies suggest Ctsb acts as a proapoptotic protease in TNF- $\alpha$ and toxic bile acid-induced cytotoxicity (13-15). This protease is released from lysosomes into the cytosol during intracellular cytotoxic-signaling cascades and triggers the mitochondrial pathway of apoptosis $(10,16)$. $\mathrm{Ctsb}$ is therefore a candidate target for inhibiting hepatocyte apoptosis in liver diseases.

Although hepatocyte apoptosis characterizes many forms of liver injury, hepatic stellate cells (HSCs) are responsible for the overabundant and maladaptive generation of matrix proteins in human liver diseases. During liver diseases, HSCs undergo a phenotypic transformation from a quiescent retinoid-storing pericyte to a myofibroblast-like cell secreting matrix proteins (17). The deposition and accumulation of matrix proteins such as type I collagen form a thick web of interconnecting fibrous scar referred to as cirrhosis. 
Although reducing hepatocyte apoptosis attenuates liver injury and inflammation, it is unclear if inhibiting apoptosis is sufficient to also abrogate stellate cell activation and liver fibrogenesis.

The aim of this study was to examine the effects of Ctsb inactivation on liver injury, inflammation, and fibrogenesis. The bile duct ligated (BDL) mouse was selected for these studies as it duplicates the hepatocyte apoptosis (12), stellate cell activation, and liver fibrosis $(18,19)$ observed in human liver diseases. To address this aim, several questions were formulated. Specifically, we asked whether Ctsb inactivation reduces (a) hepatocyte apoptosis and liver injury, (b) hepatic inflammation, and (c) hepatic fibrogenesis. The results indicate that both genetic and pharmacologic inactivation of Ctsb reduces liver injury, inflammation, and hepatic fibrogenesis during cholestasis. Because both liver injury and fibrogenesis are reduced by Ctsb inactivation, this protease is a potential target for the treatment of human liver diseases.

\section{Methods}

Extrahepatic cholestasis by ligation of the common hepatic duct. The use and the care of the animals were reviewed and approved by the Institutional Animal Care and Use Committee at the Mayo Clinic. C57BL/6 Ctsb knockout $\left(\mathrm{Ctsb}^{-/}\right)$mice and WT littermates $\left(\mathrm{Ctsb}^{+/+}\right) 6$ to 8 weeks of age were used for these studies. The generation and use of these animals has previously been described in detail (20). Common BDL was performed as previously described in detail (12). Sham-operated mice, used as controls, underwent a laparotomy with exposure, but no ligation of the common bile duct was performed.

Pharmacologic Ctsb inbibition. In selected experiments, mice were treated with the Ctsb inhibitor R-3032 (4.9 $\mathrm{mg} / \mathrm{ml}$ in 30:70 polyethylene glycol $400 / \mathrm{H}_{2} \mathrm{O}$ at $\mathrm{pH} 7.5$ ) intraperitoneally twice a day. The agent was obtained from Celera Genomics. The dose was based on pharmacokinetic data demonstrating a half-life of 4.2 hours, and preliminary data demonstrating that R-3032 extracellular concentrations of $10 \mu \mathrm{M}$ were required to maximally inhibit TNF- $\alpha$ /actinomycin D-mediated apoptosis in isolated cultured murine hepatocytes (not shown). R-3032 is a reversible Ctsb inhibitor. The $K_{\mathrm{I}}$ for Ctsb is $0.02 \mu \mathrm{M}$, whereas the $K_{\mathrm{I}}$ for cathepsin $\mathrm{K}$ is $89 \mu \mathrm{M}$, cathepsin $\mathrm{L}$ is $12 \mu \mathrm{M}$, and cathepsin $\mathrm{S}$ is 3 $\mu \mathrm{M}$. The $K_{\mathrm{I}}$ for Ctsb is at least 2 logs lower than it is for these other lysosomal cathepsin proteases, and the drug does not inhibit caspases. Thus, the drug is a selective Ctsb inhibitor.

Histology and the TUNEL assay. The liver was diced into $5 \times 5$-mm sections, fixed in $4 \%$ paraformaldehyde for 48 hours, and then embedded in paraffin (Curtin Matheson Scientific Inc., Houston, Texas, USA). Tissue sections $(4 \mu \mathrm{m})$ were prepared with a microtome (Reichert Scientific Instruments, Buffalo, New York, USA) and placed on glass slides. Hematoxylin and eosin staining was performed according to standard techniques.
TUNEL assay was performed with a commercially available kit, according to the manufacturer's instructions (In Situ Cell Death Detection Kit; Roche Diagnostics Corp., Indianapolis, Indiana, USA). Hepatocyte apoptosis in liver sections was quantitated by counting the number of TUNEL-positive cells in 30 random microscopic low-power fields $(\times 630)$.

Determination of serum total bilirubin, bile acids, and alanine aminotransferase. Total serum bilirubin, total serum bile acid, and serum alanine aminotransferase (ALT) determinations were performed using commercially available assay kits according to the manufacturer's instructions (Sigma-Aldrich Diagnostics kit no. 505, 550, and 450; Sigma-Aldrich, St. Louis, Missouri, USA).

Preparation of subcellular fractions. Cytosolic extracts (S-100) were prepared from mouse liver using the approach previously described (10). Protein concentration was measured with the Bradford assay (BioRad Laboratories Inc., Hercules, California, USA).

Immunoblot analysis. Aliquots of S-100 cytosolic extracts containing $50 \mu \mathrm{g}$ of protein were subjected to $15 \%$ SDS-PAGE and transferred to nitrocellulose membranes. Membranes were first probed overnight at $4^{\circ} \mathrm{C}$ with a mouse anti-cytochrome $c$ antibody (PharMingen, San Diego, California, USA), dilution 1:1,000, and then incubated with an HRP-conjugated goat antimouse IgG secondary antibody (BioSource International, Camarillo, California, USA) diluted 1:5,000 for 45 minutes at room temperature. Blots were developed by the enhanced chemiluminescence system (Amersham Life Sciences Inc., Arlington Heights, Illinois, USA), according to the manufacturer's instructions.

Real-time PCR. Total RNA was obtained from whole liver with the Trizol Reagent (Invitrogen Corp., Carlsbad, California, USA). For each RNA sample, a 10- $\mu \mathrm{g}$ aliquot was reverse transcribed into cDNA using oligo-dT random primers and Moloney murine leukemia virus reverse transcriptase. Real-time PCR was performed with Taq polymerase (Invitrogen Corp.) and primers for $\alpha$-smooth muscle actin ( $\alpha$-SMA), collagen $\alpha 1(\mathrm{I})$ (COL1A1), TGF- $\beta 1$, and tissue inhibitor of metalloproteinases (TIMP) as previously described (18). Primers for the experiment using inflammation were as follows: CXC chemokine ligand $1(\mathrm{KC})$, forward 5 '-TGGGATTCACCTCAAGAACA-3', reverse 5'-TGGGGACACCTTAGCATC-3' (yielding a 167-bp product); macrophage inflammatory protein-2 (MIP-2), forward 5'-CCACCAACCACCAGGCTAC- $3^{\prime}$, reverse $5^{\prime}$-GCTTCAGGGTCAAGGGCAAA- $3^{\prime}$ (221-bp); GAPDH, forward 5'-ACCCAGAAGACTGTGGATGG-3', reverse 5'-CATCGAAGGTGGAAGAGTGG-3' (340-bp). Both GAPDH and 18S primers (Ambion Inc., Austin Texas, USA ) were used as controls for RNA integrity. All PCR products were confirmed by gel electrophoresis.

Real-time PCR was performed with the LightCycler (Roche Diagnostics Corp., Mannheim, Germany) and SYBR green as the fluorophore (Molecular Probes Inc., Eugene, Oregon, USA). The result was expressed as a 

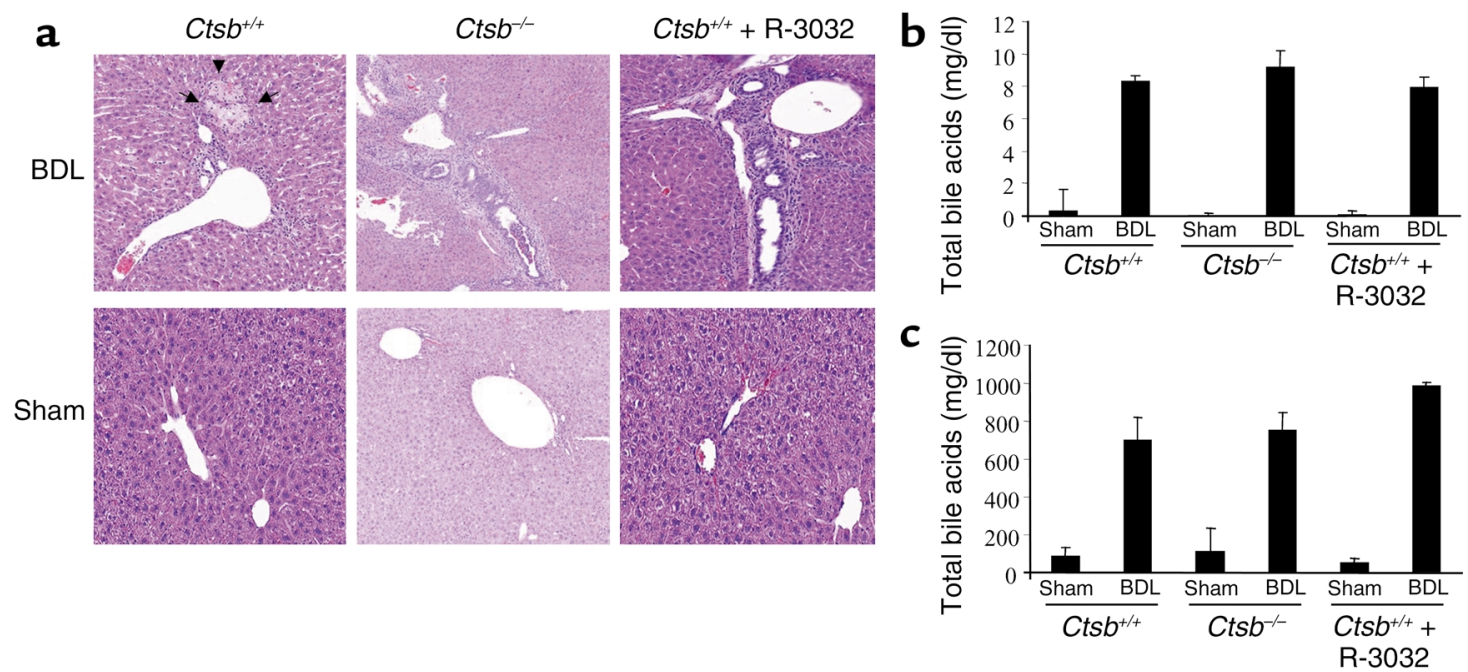

\begin{abstract}
Figure 1
The magnitude of cholestasis is similar in $\mathrm{Ctsb}^{+/+}, \mathrm{Ctsb}^{-/-}$, and R-3032-treated Ctsb $b^{+/+}$mice. Cts $b^{+/+}, \mathrm{Cts} b^{-/-}$, and R-3032-treated Ctsb $b^{+/+}$mice underwent a sham operation (control) or ligation of their common bile duct. Three days after the surgical procedure, the mice were anesthetized, and liver tissue and serum were obtained. (a) Fixed liver specimens from all mice were stained by conventional hematoxylin and eosin. Bile duct proliferation, portal edema, and mild portal infiltrates were present in all BDL mice. However, bile infarcts only occurred in $\mathrm{Ctsb}^{+/+} \mathrm{BDL}$ mice. (b and $\mathbf{c}$ ) Serum total bilirubin and total bile acids in BDL mice were comparable among the three groups.
\end{abstract}

ratio of product copies per milliliter to copies per milliliter of housekeeping gene $18 \mathrm{~S}$ and GAPDH from the same RNA (respective cDNA) sample and PCR run.

Determination of liver fibrosis. Liver fibrosis was quantified with sirius red. Direct red 80 and Fast green FCF (color index 42053) were provided by Sigma-Aldrich. The liver was diced into $5 \times 5$ - $\mathrm{mm}$ sections, immersion fixed in PBS containing 4\% paraformaldehyde for 24 hours at $4^{\circ} \mathrm{C}$, and embedded in paraffin. Ten-micrometer sections were mounted on glass slides. Sections were deparaffinized and the slides rehydrated as follows with a wash for each 5-minute step: Xylene (twice), $100 \%$ ethyl alcohol (EtOH), 95\% EtOH, 70\% EtOH, 30\% $\mathrm{EtOH}, 1 \times \mathrm{PBS}$, and distilled water (twice). The sections were incubated for 2 hours in room temperature with an aqueous solution of saturated picric acid containing $0.1 \%$ Fast green FCF and $0.1 \%$ direct red 80 . The sections were covered with aluminum foil during the incubation. Stained slides were washed slowly under running distilled water for 6 minutes, dehydrated ( 3 minutes for each step), mounted, and examined by light microscopy. Red-stained collagen fibers were quantitated by digital image analysis as described below.

The Bacus Laboratories Inc. Slide Scanner (BLISS) system (Lombard, Illinois, USA) allows for the capture and quantitation of histologic images as tiles. The tissue section on the slide was automatically scanned using a preselected magnification lens $(\times 40)$, and the captured bright-field images were digitized into a series of picture elements (pixels) each of which has a specific optical density. Through mathematical manipulation of the pixels using the instrument's software, the captured images were quantified. For sirius red, quantitation was based on determining the optical densities of the chromogen. Quantitative histomorphometry was performed on the $6-\mu \mathrm{m}-$ stained sections. The methodology was similar to that previously described $(21,22)$. Two cross sections, from two lobes from each animal were scanned. The scanned areas were captured as $\times 40$ tiles, and the evaluation by BLISS was performed without knowledge of the specimens. All images were captured with a $\times 40$ lens, the highest magnification available, as tiles with $480 \times 752$ pixel resolution. The computer automatically measured histologic features from each tile. Each captured tile was analyzed by the Histology Grade software on the instrument with separation of green and red-green-blue (RGB) filters. The red area was mathematically divided by the RGB area and multiplied by $100 \%$. This determined the percentage area staining positively for collagen fibers, providing a quantitative value on a continuous scale. The areas scanned were captured using the BLISS system. Quantitative histomorphometry was evaluated on the individual images (pixel resolution $480 \times 752$ ) for the sirius red chromogen as previously described (18).

Immunobistochemistry. The sections (5 $\mu \mathrm{m}$ thick) were stained for myeloperoxidase (MPO) using a rabbit polyclonal antibody (NeoMarkers, Fremont, California, USA), which is prediluted by the manufacturer for staining formalin-fixed, paraffin-embedded tissues. The sections were incubated with the antibody overnight at $4^{\circ} \mathrm{C}$. Negative control slides were incubated with nonimmune immunoglobulin under the same conditions. Secondary reagents were obtained from the DAKO LSAB2 kit (DAKO Corp., Carpinteria, California, USA), and 3,3'-diaminobenzidine tetrahydrochloride (Sigma-Aldrich) was used for visualization. Finally, the tissue was counterstained with hematoxylin. 
Statistics. All data represent at least four independent experiments and are expressed as the mean \pm SD unless otherwise indicated. Differences between groups were compared using ANOVA for repeated measures and a post hoc Bonferroni test to correct for multiple comparisons.

\section{Results}

Hepatocyte apoptosis and liver injury are Ctsb dependent in the BDL mouse. To examine the effects of Ctsb in mediating liver injury and hepatic fibrosis, we used $\mathrm{Cts} \mathrm{b}^{-/-}$ and $\mathrm{Ctsb}^{+/+}$littermates. Because gene-deleted animals often develop processes to compensate for the deficient alleles, selected results with $\mathrm{Cts}^{-/-}$animals were also verified by pharmacologic inhibition of Ctsb in WT littermates. Ctsb catalytic activity was inhibited using the reversible pharmacologic inhibitor R-3032. Mice were BDL for 3 days. Histopathologic examination of liver specimens demonstrated biliary proliferation, portal edema, and mild portal infiltrates in $\mathrm{Ctsb}^{+/+}, \mathrm{Ctsb}^{-/-}$, and R-3032-treated $\mathrm{Ctsb}^{+/+}$mice, indicating similar cholestatic effects of BDL in all three groups of mice (Figure 1a). Serum markers for cholestasis, total serum bilirubin, and bile acid determinations were also virtually identical in all three groups (Figure 1, b and c). In striking contrast to the comparable magnitude of cholestasis in the three experimental groups, liver cell apoptosis (TUNEL-positive cells per low-power field) was significantly reduced in $\mathrm{Cts}^{-/-}$and $\mathrm{R}-3032$-treated $\mathrm{Cts}^{+/+}$mice compared with $\mathrm{Cts} \mathrm{b}^{+/+}$mice (Figure 2, a and $b$ ). Likewise, bile infarcts (confluent foci of hepatocyte feathery degeneration caused by bile acid cytotoxicity) were only observed in BDL Ctsb ${ }^{+/+}$mice (Figure 1a). Consistent with these observations, hepatic cytosolic cytochrome $c$, suggesting activation of the mitochondrial pathway of apoptosis (23), was minimal in $\mathrm{Cts}^{-/-}$and in R-3032-treated $\mathrm{Cts}^{+/+}$animals compared with $\mathrm{Cts} b^{+/+}$BDL mice (Figure $2 \mathrm{c}$ ). Finally, serum ALT values, an index of hepatocyte injury, were also significantly lower in $\mathrm{Ctsb}^{-/-}$and R-3032-treated $\mathrm{Ctsb}^{+/+}$versus $\mathrm{Ctsb}^{+/+} \mathrm{BDL}$ mice (Figure $2 \mathrm{~d}$ ). The ALT elevations in the BDL WT mice may be secondary to apoptosis and/or to the associated inflammatory response that occurs in this liver disease model (see below). These data implicate a pathogenic role for Ctsb in cholestatic liver injury.

Hepatic inflammation is Ctsb dependent in the BDL mouse. Hepatocyte apoptosis is a proinflammatory process in the liver that promotes chemokine generation and a

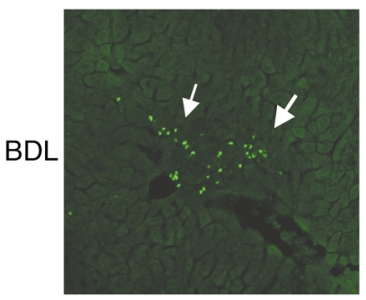

b

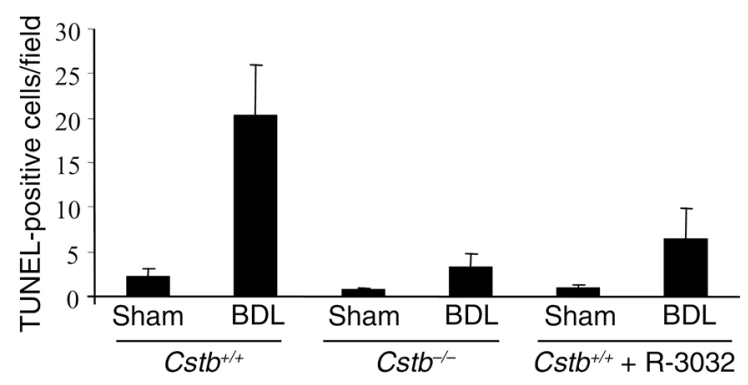

Cstb-1-
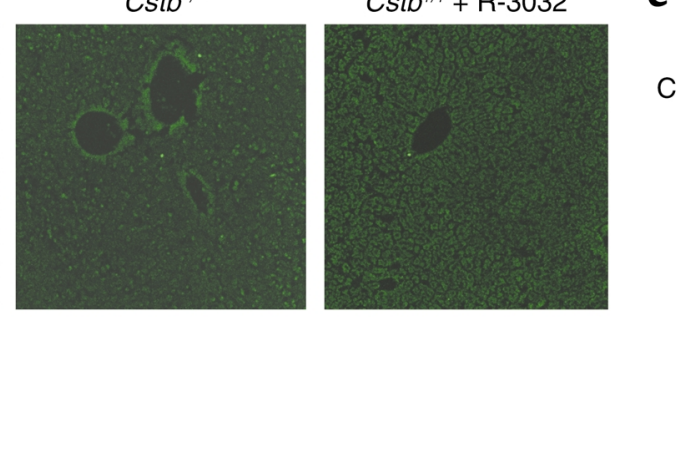

d

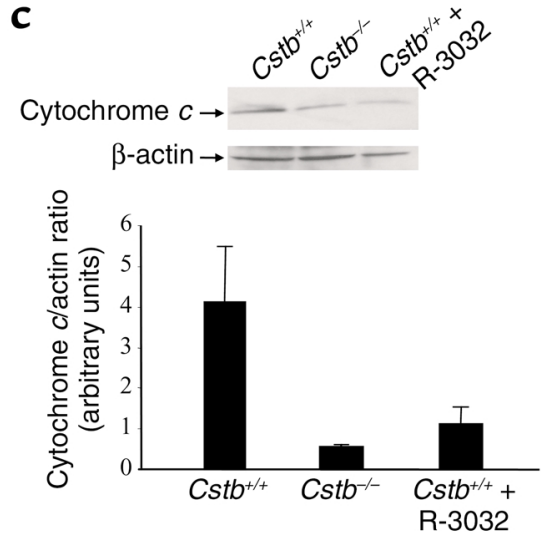

$\mathrm{R}-3032$

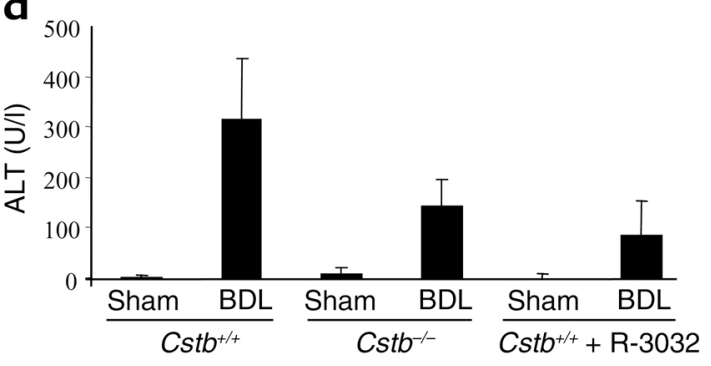

\section{Figure 2}

Liver injury and cytochrome $c$ release are reduced in $\mathrm{Ctsb}^{-/-}$and R-3032-treated $\mathrm{Cts} b^{+/+}$mice. Ctsb $b^{+/+}$and $\mathrm{Ctsb} b^{-/-}$mice were BDL for 3 days as described in Methods. (a) Fixed liver specimens were analyzed by TUNEL assay to identify apoptotic hepatocytes (arrows). (b) The number ofTUNEL-positive cells was significantly higher in $\mathrm{Ctsb}^{+/+} \mathrm{BDL}$ mice than in sham-operated control or Ctsb $b^{-/-}$and R-3032-treated Ctsb $b^{+/} \mathrm{BDL}$ mice $(P<0.0001, n=4$ for each experimental group). (c) Cytosolic and mitochondrial fractions were prepared as described in Methods. Aliquots of $50 \mu \mathrm{g}$ of cytosolic protein were subjected to SDS-PAGE and immunoblotted for cytochrome $c$. Release of cytochrome $c$ in the cytosol was higher in $\mathrm{Ctsb}^{+/+} \mathrm{BDL}$ livers compared with $\mathrm{Cts}^{-/-}$and R-3032-treated $\mathrm{Cts} b^{+/+} \mathrm{BDL}$ mice. A representative immunoblot from one animal from each group is shown, together with densitometric analysis of multiple immunoblots ( $n=3$ animals for each group). (d) Serum $\mathrm{ALT}$ values are significantly greater in $\mathrm{Cts}^{+/+}$than in $\mathrm{Ctsb}^{-/-}\left(P<0.005, n=4\right.$ for each experimental group) and R-3032-treated $\mathrm{Ctsb}^{+/+}$mice 3 days after $\mathrm{BDL}(P<0.0001, n=4$ for each experimental group). $U / I$, units per liter. 

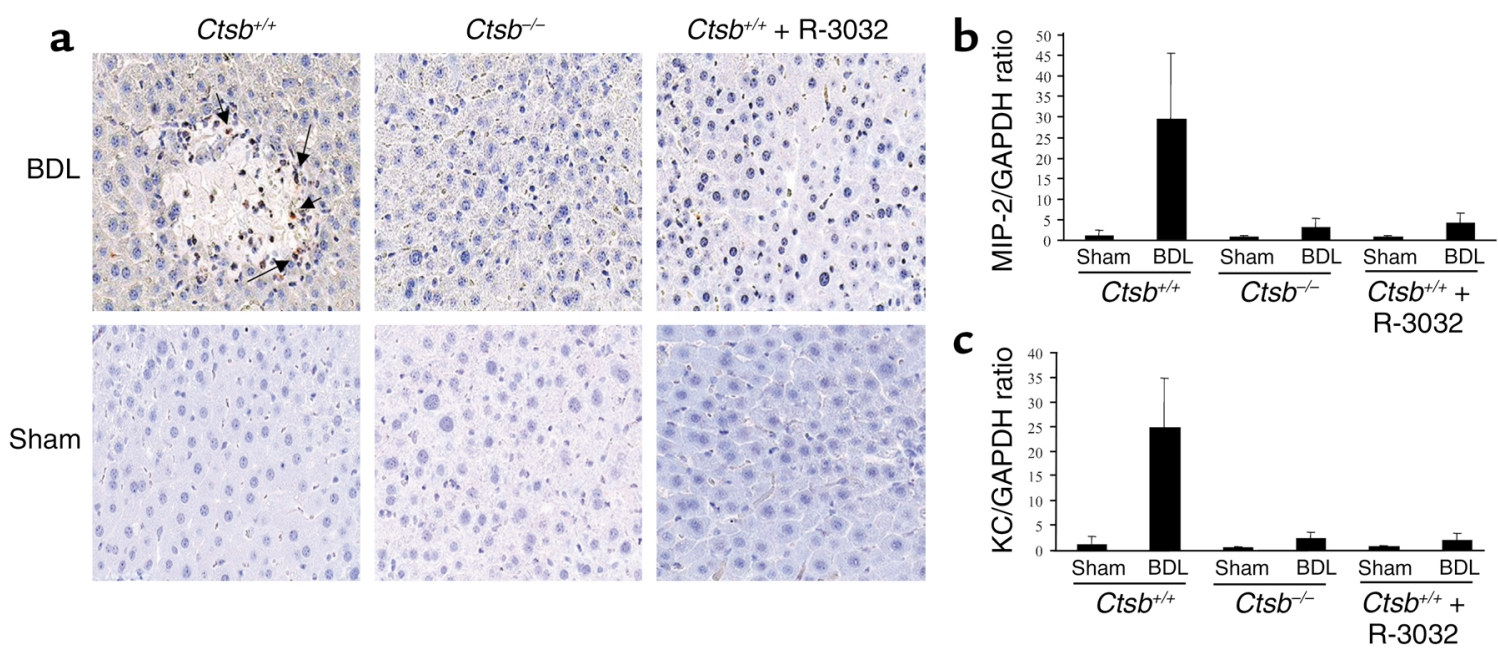

\begin{abstract}
Figure 3
Neutrophilic infiltration and chemokine expression are increased in $\mathrm{Cts} b^{+/+}$compared with $\mathrm{Cts} b^{-/-}$and R-3032-treated Ctsb $b^{+/+} \mathrm{BDL}$ mice. Fixed liver specimens from mice were stained for MPO, a neutrophil marker. Neutrophil infiltration was present only in $\mathrm{Cts} b^{+/+} \mathrm{BDL}$ mice $(\mathbf{a})$ (original magnification $\times 20$ ). Three days after the surgical procedure, liver tissue was procured and total hepatic RNA was isolated as described in Methods. (b and $\mathbf{c}$ ) MIP-2 and KC expression was quantitated by real-time PCR. The expression was normalized as a ratio using GAPDH mRNA as a housekeeping gene. A value of 1 for this ratio was arbitrarily assigned to the data obtained from sham-operated $\mathrm{Cts} b^{+/+}$ mice. KC mRNA expression in $\mathrm{Ctsb}^{+/+} \mathrm{BDL}$ was higher than in $\mathrm{Cts}^{-/-}(P<0.0001)$ and R-3032-treated $\mathrm{Cts} b^{+/+} \mathrm{BDL}$ mice $(P<0.0001, n=4$ for each group). The expression of MIP-2 mRNA was greater in $\mathrm{Cts}^{+/+}$than in $\mathrm{Cts}^{-/-}(P<0.0001)$ and R-3032-treated $\mathrm{Ctsb}^{+/+} \mathrm{BDL}$ mice $(P<0.0001, n=4$ for each group).
\end{abstract}

neutrophil infiltration (6-8). To examine the effects of Ctsb in mediating liver inflammation, we used BDL $\mathrm{Cts}^{-/-}$and $\mathrm{Cts} b^{+/+}$littermates and verified the observations by pharmacologic inhibition of Ctsb in BDL WT littermates. Mice were BDL for 3 days. Immunohistochemistry examination of liver specimens for MPO (a marker for neutrophils) demonstrated neutrophil infiltrates in BDL Ctsb $b^{+/+}$, but not in BDL $\mathrm{Ctsb}^{-/-}$or in R-3032-treated BDL Ctsb ${ }^{+/+}$animals (Figure 3a). To investigate the role of Ctsb-mediated hepatic chemokine expression, mRNA was extracted from the livers of $\mathrm{Ctsb}^{-/-}$, R-3032-treated $\mathrm{Ctsb}^{+/+}$, and $\mathrm{Ctsb}^{+/+}$mice that had undergone BDL for 3 days. KC and MIP-2 mRNA transcripts, neutrophil chemoattractants, were quantitated using real-time PCR technology $(4,24,25)$. In $\mathrm{Ctsb}^{+/+}$animals BDL for 3 days, the mRNA for these two transcripts were increased 24-fold or greater compared with sham-operated controls (Figure 3, b and c). More importantly, the transcripts for KC and MIP-2 were significantly reduced in $\mathrm{Cts}^{-/-}$and R-3032-treated $\mathrm{Cts}^{+/+} \mathrm{BDL}$ animals compared with $\mathrm{Cts}^{+/+} \mathrm{BDL}$ mice (Figure 3, b and c). These data suggest neutrophil-associated inflammation occurs in cholestasis and is coupled to Ctsb-associated liver injury.

Liver fibrogenesis is $C t s b$ dependent in the BDL mouse. To investigate the role of Ctsb-mediated liver injury in liver fibrogenesis, mRNA was extracted from the livers of $\mathrm{Cts}^{-/-}, \mathrm{R}-3032$-treated $\mathrm{Cts}^{+/+}$, and $\mathrm{Cts} \mathrm{b}^{+/+}$mice that had undergone BDL for 3 days. The $\alpha$-SMA mRNA transcripts reflecting HSC activation were quantitated using real-time PCR technology. In $\mathrm{Ctsb}^{+/+}$animals BDL for 3 days, the mRNA for this transcript was increased compared with that in sham-operated controls, indicating stellate cell activation in this model of cholestasis (Figure 4a). The transcripts for $\alpha$-SMA were significantly reduced in $\mathrm{Ctsb}^{-/-}$and R-3032-treated $\mathrm{Cts}^{+/+} \mathrm{BDL}$ animals compared with $\mathrm{Cts} b^{+/+} \mathrm{BDL}$ mice (Figure 4a). These data suggest HSC activation in cholestasis is, in part, Ctsb dependent.

To ascertain if the observed indicators of HSC activation were also associated with fibrogenesis (17), transcripts for molecules implicated in fibrogenesis were quantitated. TGF- $\beta$ mRNA, a cytokine playing an instrumental role in liver fibrogenesis (26), was greater

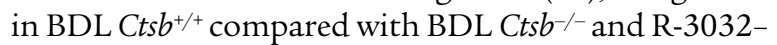
treated BDL Ctsb $b^{+/+}$mice (Figure 4b). COL1A1 mRNA expression was also markedly increased in BDL $\mathrm{Cts}^{+/+}$ versus $\mathrm{Ctsb}^{-/-}$and R-3032-treated $\mathrm{BDL} \mathrm{Ctsb}^{+/+}$mice (Figure 4c). In chronic liver injury, activated stellate cells are the major source of tissue inhibitors of metalloproteinases (TIMPs), which inhibit collagen degradation by MMPs and protect HSCs from apoptosis (27-29). However, compared with the increases in $\alpha$-SMA, TGF- $\beta$, and COL1A1, alterations in TIMP mRNA were modest and not significantly different between the experimental groups (Figure 4d). Collectively, these data suggest that Ctsb-mediated liver injury is associated with HSC activation and hepatic fibrogenesis.

Hepatic collagen deposition is $C t s b$ dependent in the BDL mouse. To examine the relationship between Ctsbmediated liver injury and fibrosis, mice were BDL and $\mathrm{Cts} b^{+/+}$mice were treated with R-3032 intraperitoneally twice a day for 2 weeks. This duration of BDL was necessary for the development of septal fibrosis as assessed by classic histopathologic techniques. Hepatic collagen deposition/accumulation 

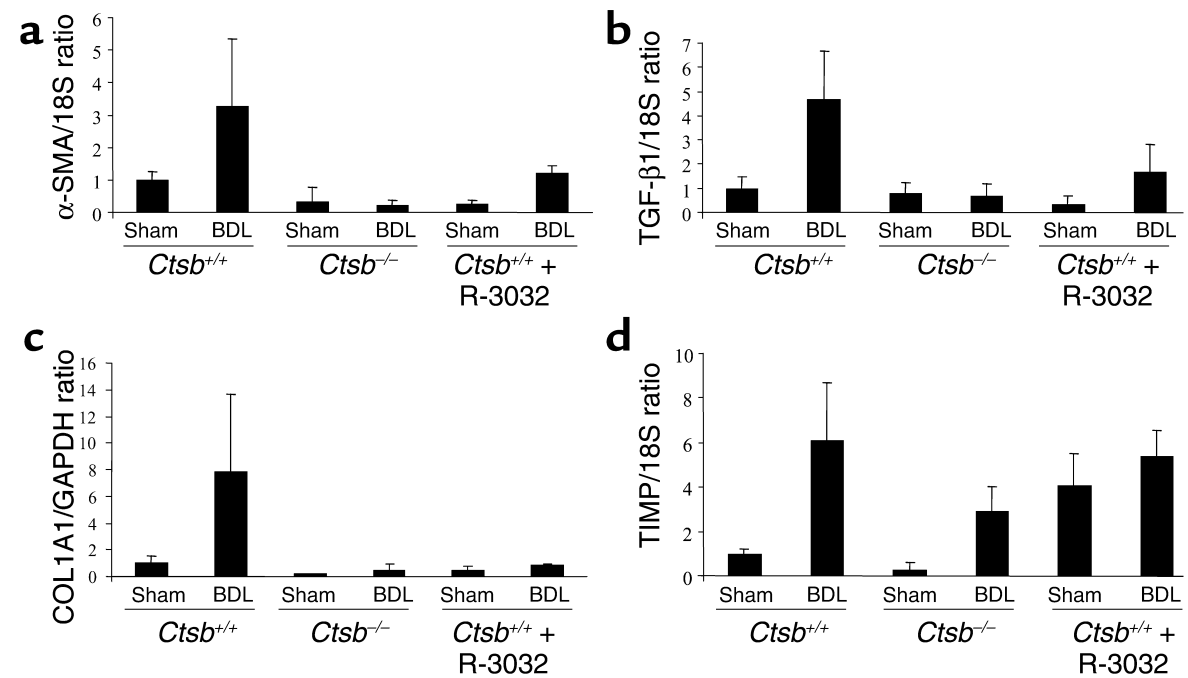

\begin{abstract}
Figure 4
Markers for $\mathrm{HSC}$ activation are increased in $\mathrm{Ctsb}^{+/+}$compared with $\mathrm{Ctsb}^{-/-}$and R-3032-treated Ctsb $b^{+/+} \mathrm{BDL}$ mice. Three days after the surgical procedure, liver tissue was procured and total hepatic RNA was isolated as described in Methods. $\alpha-S M A, T G F-\beta 1, C O L 1 A 1$, and TIMP were quantitated by real-time PCR. The expression was normalized as a ratio using $18 \mathrm{~S}$ and GAPDH mRNA as housekeeping genes. A value of 1 for this ratio was arbitrarily assigned to the data obtained from sham-operated $C t s b^{+/+}$mice. (a) $\alpha$-SMA mRNA expression in $C t s b^{+/+} \mathrm{BDL}$ was higher than in $\mathrm{Ctsb}^{-/-}(P<0.0001)$ and R-3032-treated Ctsb ${ }^{+/+}$BDL mice $(P<0.0003, n=4$ for each group $)$. (b) The expression of TGF- $\beta 1 \mathrm{mRNA}$ was greater in $\mathrm{Ctsb}^{+/+}$than in $\mathrm{Cts} b^{-/-}(P<0.0001)$ and R-3032-treated $C t s b^{+/+} \mathrm{BDL}$ mice $(P<0.0001, n=4$ for each group). (c) The expression of COL1A1 mRNA was significantly elevated in $\mathrm{Cts}^{+/+} \mathrm{BDL}$ mice and markedly attenuated in $\mathrm{Cts} b^{-/-}(P<0.0001)$ and R-3032-treated $\mathrm{Cts} b^{+/+}$ BDL mice $\left(P<0.0001, n=4\right.$ for each group). (d) The expression of TIMP mRNA was not significantly different in Cts $b^{+/+}, C_{s} b^{-/-}$, and R-3032treated $\mathrm{Cts}^{+/+} \mathrm{BDL}$ mice ( $P=\mathrm{NS}, n=4$ for each group).
\end{abstract}

was stained with sirius red (Figure 5a) and quantitated using digital image analysis (Figure $5 \mathrm{~b}$ ). Significant collagen staining was present in mice that had undergone BDL for 2 weeks; however, the quantity of collagen was again greater in $\mathrm{Ctsb}^{+/+}$than in $\mathrm{Ctsb}^{-/-}$ and in R-3032-treated Ctsb ${ }^{+/+}$BDL mice (Figure 5, a and $\mathrm{b}$ ). Taken together, these observations suggest Ctsb-mediated liver injury during extrahepatic cholestasis results in fibrogenesis and collagen deposition within the liver.

\section{Discussion}

The principal findings of this study relate Ctsb inactivation to liver injury and fibrogenesis in cholestasis. The observations demonstrate that, in the BDL animal, both genetic and pharmacologic inactivation of Ctsb reduces (a) hepatocyte apoptosis, serum ALT values, and histologic evidence of liver injury; (b) hepatic inflammation, as assessed by expression of chemoattractants and neutrophil infiltration; (c) mRNA expression for markers of HSC fibrogenic

\section{Figure 5}

Hepatic fibrosis is increased in $\mathrm{Cts}^{+/+} \mathrm{BDL}$ compared with $\mathrm{Cts}^{-/-} \mathrm{BDL}$ mice. (a) Two weeks after the surgical procedure, liver tissue was obtained from BDL and sham-operated $\mathrm{Ctsb}^{+/+}, \mathrm{Ctsb}^{-/-}$, and R-3032-treated $\mathrm{Cts}^{+/+} \mathrm{BDL}$ mice, and collagen fibers were stained with sirius red as described in Methods. (b) The surface area stained with sirius red was quantitated using digital image analysis. Sirius red staining was quantitatively greater in $\mathrm{Ctsb}^{+/+} \mathrm{BDL}$ than in $\mathrm{Ctsb}^{-/-}$and R-3032treated $\mathrm{Cts}^{+/+} \mathrm{BDL}$ mice $(P<0.001, n=4$ for each group). Only minimal sirius red staining was observed in sham-operated mice from the three groups of animals. (Original magnification $\times 20$.) $\mathbf{a}$
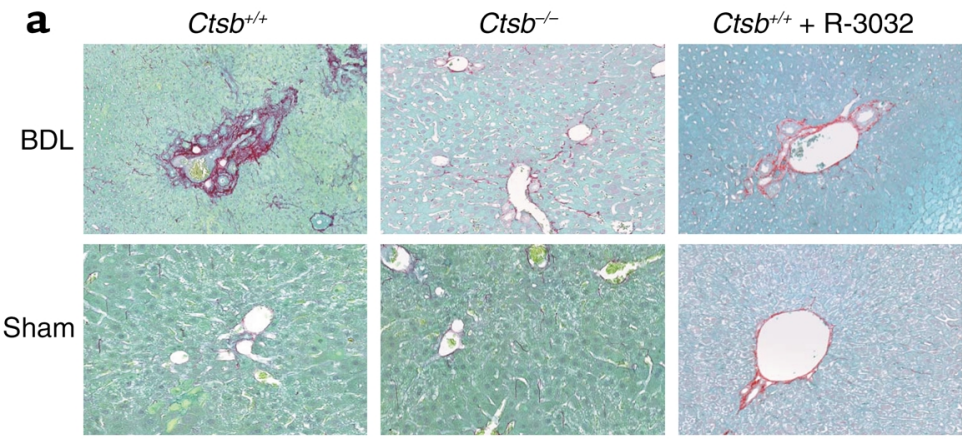

b

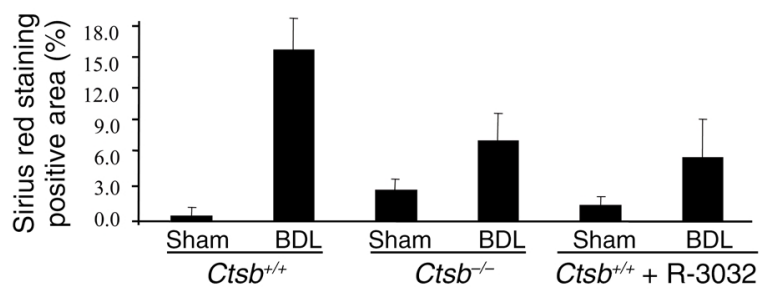


activity; and (d) collagen deposition. Taken together, these observations suggest a critical role for this protease in mediating the deleterious consequences of cholestasis. Each of these observations is discussed in greater detail below.

Increasing evidence implicates Ctsb as a proapoptotic protease. For example, Ctsb contributes to bile saltand TNF- $\alpha$-induced hepatocyte apoptosis $(10,14,30$, 31 ), and pharmacologic inhibition of Ctsb blocks apoptosis induced by p53 and cytotoxic agents (32). Ctsb activity appears to play a critical role in lysosomal permeabilization in cytotoxic events, causing release of lysosomal proteases into the cytosol, which in turn cause mitochondrial dysfunction $(10,16)$. The current findings extend these observations by demonstrating a role for this protease in vivo during a model of human liver disease, namely, cholestasis. Consistent with the prior in vitro studies, Ctsb would appear to contribute to hepatocyte apoptosis by causing mitochondrial dysfunction and cytochrome $c$ release. Indeed, cytosolic cytochrome $c$ was markedly reduced in BDL $\mathrm{Cts}^{-/-}$and in R-3032-treated $\mathrm{Ctsb}^{+/}$animals compared with BDL WT animals. How Ctsb contributes to mitochondrial dysfunction remains to be elucidated, but our previous in vitro studies implicated a requirement for additional cytosolic factor(s) (10). Nonetheless, the current studies provide mechanistic evidence implicating the lysosomal protease Ctsb and therefore a dominant role for the lysosomal pathway of hepatocyte apoptosis in cholestatic liver injury.

Our study also shows that Ctsb-mediated liver injury not only causes apoptosis but also stimulates production of proinflammatory chemokines. Hepatocyte apoptosis therefore appears to be associated with the generation of chemokines promoting inflammation in cholestasis. Indeed, in other models of liver injury hepatocyte apoptosis has also been linked to inflammation. For example, Fas-mediated hepatocyte apoptosis elicits an inflammatory response in the liver that could secondarily induce HSC activation $(25,33)$. Although originally thought of as a "silent process," unorchestrated and continuous apoptosis in the liver is likely profibrogenic in pathophysiologic conditions.

Our studies suggest a role for Ctsb in hepatic fibrogenesis during cholestasis. Ctsb could have a direct or indirect effect on hepatic fibrogenesis. It is likely that Ctsb inactivation and consequent attenuation of hepatocyte apoptosis is the mechanism linking this proapoptotic protease to liver fibrogenesis. Indeed, inhibition of Fas-mediated apoptosis also attenuates hepatic fibrogenesis, supporting a link between liver cell apoptosis and HSC activation $(18,19)$. The mechanism by which hepatocyte apoptosis results in hepatic fibrogenesis is likely indirect. Hepatocyte apoptosis elicits an inflammatory response associated with chemokine expression and neutrophil infiltration (5-8). This inflammatory response has been well established to cause stellate cell activation (34). The activated stellate cells produce collagen, causing liver scarring. Alternatively, engulfment of hepatocyte apoptotic bodies directly by stellate cells may also promote activation of these cells as has been observed in vitro (35). However, based on current concepts, we favor the following model linking Ctsb-associated hepatocyte apoptosis to inflammation and stellate cell activation. Ctsb, via cytotoxic signaling cascades initiated by toxic bile acids or perhaps TNF- $\alpha$, induces hepatocyte apoptosis. This injurious process promotes hepatic inflammatory responses. The inflammation subsequently induces stellate cell activation and fibrogenesis. This linear model (apoptosis $\rightarrow$ inflammation $\rightarrow$ fibrosis) is likely simplistic but provides a framework to further study apoptosis as an inciting event in liver injury and scarring.

In summary, our findings suggest that during extrahepatic cholestasis in the mouse, liver injury, inflammation, markers of stellate cell activation, and elevation of indices of hepatic fibrogenesis are, in part, Ctsb dependent. Furthermore, inactivation of Ctsb catalytic activity with selective protease inhibitors such as R-3032 indicates a potential therapeutic option for cholestatic liver injury, inflammation, and fibrosis. These data also implicate a mechanistic link between hepatocyte apoptosis and HSC activation and suggest inhibition of apoptosis would diminish liver fibrosis in chronic cholestatic liver diseases. These concepts merit further investigation as mechanism(s) contributing to the development of cirrhosis and as potential antifibrotic therapeutic strategies.

\section{Acknowledgments}

This work was supported by a fellowship grant from the Postdoctoral Program of the German Academic Exchange Service to A. Canbay, NIH grant DK063947 to G.J. Gores, and the Mayo Foundation. The secretarial assistance of Erin Bungum is gratefully acknowledged. We thank Darren L. Riehle and James Tarara for their excellent technical assistance in digital image analysis.

1. Kim, W.R., Brown, R.S., Jr., Terrault, N.A., and El-Serag, H. 2002. Burden of liver disease in the United States: summary of a workshop. Hepatology. 36:227-242.

2. Sandler, R.S., et al. 2002. The burden of selected digestive diseases in the United States. Gastroenterology. 122:1500-1511.

3. Galle, P.R., et al. 1995. Involvement of the CD95 (APO-1/Fas) receptor and ligand in liver damage. J. Exp. Med. 182:1223-1230.

4. Luster, A.D. 1998. Chemokines: chemotactic cytokines that mediate inflammation. N. Engl. J. Med. 338:436-445.

5. Miwa, K., et al. 1998. Caspase 1-independent IL-1beta release and inflammation induced by the apoptosis inducer Fas ligand. Nat. Med. 4:1287-1292.

6. Lawson, J.A., Fisher, M.A., Simmons, C.A., Farhood, A., and Jaeschke, H. 1998. Parenchymal cell apoptosis as a signal for sinusoidal sequestration and transendothelial migration of neutrophils in murine models of endotoxin and Fas-antibody-induced liver injury. Hepatology. 28:761-767.

7. Jaeschke, H. 2002. Inflammation in response to hepatocellular apoptosis. Hepatology. 35:964-966.

8. Maher, J.J., Scott, M.K., Saito, J.M., and Burton, M.C. 1997. Adenovirusmediated expression of cytokine-induced neutrophil chemoattractant in rat liver induces a neutrophilic hepatitis. Hepatology. 25:624-630.

9. Leist, M., Gantner, F., Jilg, S., and Wendel, A. 1995. Activation of the $55 \mathrm{kDa}$ TNF receptor is necessary and sufficient for TNF-induced liver failure, hepatocyte apoptosis, and nitrite release. J. Immunol. 154:1307-1316. 
10. Guicciardi, M.E., et al. 2000. Cathepsin B contributes to TNF-alphamediated hepatocyte apoptosis by promoting mitochondrial release of cytochrome c. J. Clin. Invest. 106:1127-1137.

11. Faubion, W.A., et al. 1999. Toxic bile salts induce rodent hepatocyte apoptosis via direct activation of Fas. J. Clin. Invest. 103:137-145.

12. Miyoshi, H., Rust, C., Roberts, P.J., Burgart, L.J., and Gores, G.J. 1999. Hepatocyte apoptosis after bile duct ligation in the mouse involves Fas. Gastroenterology. 117:669-677.

13. Guicciardi, M.E., Miyoshi, H., Bronk, S.F., and Gores, G.J. 2001. Cathep$\sin \mathrm{B}$ knockout mice are resistant to tumor necrosis factor-alpha-mediated hepatocyte apoptosis and liver injury: implications for therapeutic applications. Am. J. Pathol. 159:2045-2054.

14. Roberts, L.R., et al. 1997. Cathepsin B contributes to bile salt-induced apoptosis of rat hepatocytes. Gastroenterology. 113:1714-1726.

15. Luo, X., Budihardjo, I., Zou, H., Slaughter, C., and Wang, X. 1998. Bid, a $\mathrm{Bcl} 2$ interacting protein, mediates cytochrome $\mathrm{c}$ release from mitochondria in response to activation of cell surface death receptors. Cell. 94:481-490.

16. Werneburg, N.W., Guicciardi, M.E., Bronk, S.F., and Gores, G.J. 2002. Tumor necrosis factor-alpha-associated lysosomal permeabilization is cathepsin B dependent. Am. J. Physiol. Gastrointest. Liver Physiol. 283:G947-G956.

17. Friedman, S.L. 2000. Molecular regulation of hepatic fibrosis, an integrated cellular response to tissue injury. J. Biol. Chem. 275:2247-2250.

18. Canbay, A., et al. 2002. Fas enhances fibrogenesis in the bile duct ligated mouse: a link between apoptosis and fibrosis. Gastroenterology. 123:1323-1330.

19. Song, E., et al. 2003. RNA interference targeting Fas protects mice from fulminant hepatitis. Nat. Med. 9:347-351.

20. Deussing, J., et al. 1998. Cathepsins B and D are dispensable for major histocompatibility complex class II-mediated antigen presentation. Proc. Natl. Acad. Sci. U. S. A. 95:4516-4521.

21. Sebo, T.J. 1995. Digital image analysis. Mayo Clin. Proc. 70:81-82.

22. Boone, C.W., et al. 2000. Quantitative grading of rat esophageal carcino- genesis using computer-assisted image tile analysis. Cancer Epidemiol. Biomarkers Prev. 9:495-500.

23. Green, D.R., and Reed, J.C. 1998. Mitochondria and apoptosis. Science. 281:1309-1312.

24. Bajt, M.L., Farhood, A., and Jaeschke, H. 2001. Effects of CXC chemokines on neutrophil activation and sequestration in hepatic vasculature. Am. J. Physiol. Gastrointest. Liver Physiol. 281:G1188-G1195.

25. Faouzi, S., et al. 2001. Anti-Fas induces hepatic chemokines and promotes inflammation by an NF-kappa B-independent, caspase-3-dependent pathway. J. Biol. Chem. 276:49077-49082.

26. Hellerbrand, C., Stefanovic, B., Giordano, F., Burchardt, E.R., and Brenner, D.A. 1999. The role of TGFbeta1 in initiating hepatic stellate cell activation in vivo. J. Hepatol. 30:77-87.

27. Iredale, J.P. 2001. Hepatic stellate cell behavior during resolution of liver injury. Semin. Liver Dis. 21:427-436.

28. Iredale, J.P., et al. 1998. Mechanism of spontaneous resolution of rat liver fibrosis. J. Clin. Invest. 102:538-549.

29. Murphy, F.R., et al. 2002. Inhibition of apoptosis of activated hepatic stellate cells by tissue inhibitor of metalloproteinase- 1 is mediated via effects on matrix metalloproteinase inhibition: implications for reversibility of liver fibrosis. J. Biol. Chem. 277:11069-11076.

30. Roberts, L.R., Adjei, P.N., and Gores, G.J. 1999. Cathepsins as effector proteases in hepatocyte apoptosis. Cell Biochem. Biophys. 30:71-88.

31. Foghsgaard, L., et al. 2001. Cathepsin B acts as a dominant execution protease in tumor cell apoptosis induced by tumor necrosis factor. J. Cell Biol. 153:999-1010.

32. Yuan, X.M., et al. 2002. Lysosomal destabilization in p53-induced apoptosis. Proc. Natl. Acad. Sci. U. S. A. 99:6286-6291.

33. Jaeschke, H., et al. 2002. Mechanisms of hepatotoxicity. Toxicol. Sci. 65:166-176.

34. Maher, J.J. 2001. Interactions between hepatic stellate cells and the immune system. Semin. Liver Dis. 21:417-426.

35. Canbay, A., et al. 2003. Apoptotic body engulfment by a human stellate cell line is profibrogenic. Lab. Invest. 83:655-663. 